\title{
On Optimal Regularization Methods for the Backward Heat Equation
}

\author{
U. Tautenhahn and T. Schröter
}

\begin{abstract}
In this paper we consider different regularization methods for solving the heat equation $u_{t}+A u=0 \quad(0 \leq t<T)$ backward in time, where $A: H \rightarrow H$ is a linear (unbounded) operator in a Hilbert space $H$ with norm $\|\cdot\|$ and $z^{\delta}$ are the available (noisy) data for $u(T)$ with $\left\|z^{\delta}-u(T)\right\| \leq \delta$. Assuming $\|u(0)\| \leq E$ we consider different regularized solutions $q_{\alpha}^{\delta}(t)$ for $u(t)$ and discuss the question how to choose the regularization parameter $\alpha=\alpha(\delta, E, t)$ in order to obtain optimal estimates sup $\left\|q_{\alpha}^{\delta}(t)-u(t)\right\| \leq E^{1-t} \delta$ where the supremum is taken over $z^{\delta} \in H,\|u(0)\| \leq E$ and $\left\|z^{\delta}-u(T)\right\| \leq \delta$.
\end{abstract}

Keywords: Backward heat equation, optimal parameter choice, optimal error bounds, regularization methods

AMS subject classification: Primary $65 \mathrm{M} 30$, secondary $35 \mathrm{R} 25$

\section{Introduction}

In this paper we consider different regularization methods for solving the heat equation backward in time in which the temperature $q(t)=u(x, t) \quad(t \in[0, T))$ has to be determined while temperature data $z(x)=u(x, T)$ are given and $u(x, t)$ satisfies the evolution equation

$$
u_{t}+\dot{A} u=0 \quad(0 \leq t<T)
$$

with a linear, densely defined, self adjoint and positive definite operator $A: D(A) \subseteq$ $H \rightarrow H$ with eigenvalues $\lambda_{i}(i \in \mathbb{N})$ :

$$
0<\lambda_{1} \leq \lambda_{2} \leq \ldots \quad \text { with } \quad \lambda_{i} \rightarrow \infty \text { for } i \rightarrow \infty
$$

and eigenelements $u_{i}(i \in \mathbb{N})$ that form an orthonormal basis in a real Hilbert space $H$ with norm $\|\cdot\|$ and inner product $(\cdot, \cdot)$. One simple example for $(1.1)$ is the onedimensional heat equation

$$
\left.\begin{array}{rl}
u_{t}-u_{x x}=0 & \text { for }(x, t) \in(0, \pi) \times(0, T) \\
u(0, t)=u(\pi, t)=0 \quad \text { for } t \in[0, T],
\end{array}\right\}
$$

U. Tautenhahn: Hochschule für Techn., Wirtsch. u. Sozialwesen Zittau/Görlitz, Dep. Math., P.O. Box 261, D - 02763 Zittau

T. Schröter: Techn. Univ. Chemnitz-Zwickau, Fat. Math., D - 09107 Chemnitz 
in which the eigenvalues $\lambda_{i}$ and eigenelements $u_{i}$ of $A: H_{0}^{1}(0, \pi) \cap H^{2}(0, \pi) \subset H \rightarrow H$ with $H=L^{2}(0, \pi)$ are given by

$$
\lambda_{i}=i^{2} \quad \text { and } \quad u_{i}=\sqrt{\frac{2}{\pi}} \sin i x \quad(i \in \mathbb{N})
$$

respectively.

Let us formulate the above backward heat equation problem as an operator equation

$$
K(t) q(t)=z
$$

By Fourier method one has the usual 'forward solution"

$$
u(x, t)=\sum_{i=1}^{\infty}\left(q(0), u_{i}\right) e^{-\lambda_{i} t} u_{i}
$$

of problem (1.1), consequently, $\left(q(0), u_{i}\right)=\left(q(t), u_{i}\right) e^{\lambda_{i} t}=\left(q(T), u_{i}\right) e^{\lambda_{i} T}$, which gives $\left(q(T), u_{i}\right)=\left(q(t), u_{i}\right) e^{-\lambda_{i}(T-t)}$ and shows us that for any fixed $t \in[0, T)$ the operator $K(t): H \rightarrow H$ of problem (1.3) has the representation

$$
K(t) q(t)=\sum_{i=1}^{\infty}\left(q(t), u_{i}\right) e^{-\lambda_{i}(T-t)} u_{i}
$$

Consequently, $K(t): H \rightarrow H$ is a linear selfadjoint compact operator with eigenvalues $\kappa_{i}(t)=e^{-\lambda_{i}(T-t)}$ and eigenelements $u_{i}$. Since the eigenvalues $\kappa_{i}(t)$ of the operator $K(t)$ decay exponentially fast we realize that problem (1.3) is a severely ill-posed problem. The ill-posedness becomes worse as $t$ decreases. The numerical treatment of linear illposed problems in which the solution $q(t)$ of problem (1.3) does not depend continuously on the data requires the application of special regularization methods. For the general regularization theory for linear ill-posed problems we refer to $[1,2,5,6,8,11,18$, 21]; special regularization methods for the heat equation backward in time have been considered, e.g. in $[3,7,10,13,14,17)$.

We suppose that instead of $z=u(T)$ noisy data $z^{\delta} \in H$ are available and assume throughout this paper that

(A1) $\left\|z-z^{\delta}\right\| \leq \delta$

(A2) $\|q(0)\| \leq E$

with known bounds $\delta$ and $E$. Let, for any fixed $t<T, q(t)$ the solution of problem (1.3). Then, since $q(t)$ can be represented by

$$
q(t)=\sum_{i=1}^{\infty} \frac{\left(z, u_{i}\right)}{\kappa_{i}(t)} u_{i}=\sum_{i=1}^{\infty} \frac{\left(z, u_{i}\right)}{\kappa_{i}(0)} \kappa_{i}(t)^{\frac{1}{\tau-i}} u_{i}
$$

we obtain that

$$
q(t)=K(t)^{p} q(0) \quad \text { with } \quad p=\frac{t}{T-t}
$$


From the source representation (1.5) for the exact solution $q(t)$ of:problem (1.3) we realize that (for any fixed $t \in[0, T)$ ) condition (A2) can be reformulated into the condition $q(t) \in M_{p, E}$ where the source set $M_{p, E}$ is given by

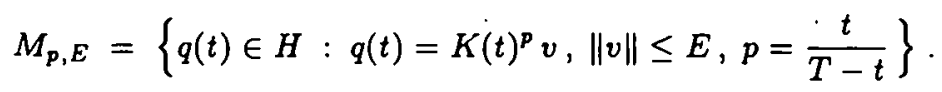

Any operator $R(t): H \rightarrow H$ can be considered as a special method for identifying the solution $q(t)$ of problem (1.3) from noisy data $z^{\delta} \in H$; the approximate solution to (1.3) is then given by $R(t) z^{\delta}$. We introduce the worst.case error $\Delta(\delta, R(t))$ for.identifying $q(t)$ from $z^{\delta} \in H$ under the conditions $\left\|z-z^{\delta}\right\| \leq \delta$ and $q(t) \in M_{p, E}$ by

$$
\Delta(\delta, R(t))=\sup _{\substack{\delta(z) \in M_{p, E}, z^{\delta} \in B \\\left\|K(1)_{q}(1)-z^{\delta}\right\| \leq \delta}}\left\|R(t) z^{\delta}-q(t)\right\|
$$

where the side condition $q(t) \in M_{p, E}$ can be replaced by the equivalent condition (A2). This worst case error characterizes the maximal error of an arbitrary method $R(t)$ if the solution $q(t)$ varies in the set $M_{p, E}$. Now we ask the question concerning the magnitude of the worst case error $\Delta(\delta, R(t))$ for 'optimal' methods $R(t): H \rightarrow H$. From [21] we have the result that

$$
\inf _{R(t)} \Delta(\delta, R(t)) \geq E^{\frac{1}{p+1}} \delta^{\frac{p}{p+1}}=E^{1-\frac{t}{T}} \delta^{\frac{t}{T}}
$$

(where the infimum is taken over all methods $R(t): H \rightarrow H$ ) provided $\frac{\delta}{E} \in \sigma\left(K(t)^{p+1}\right.$ ) where $\sigma(K(t))$ denotes the spectrum of mapping $K(t)$. Note that condition $\frac{\delta}{E} \epsilon$ $\sigma\left(K(t)^{p+1}\right)$ can only hold (for sufficiently small $\delta$ ) provided problem (1.3) is ill-posed, which means in the compact selfadjoint case that the eigenvalues $\kappa_{i}(t)$ tend to zero. For well-posed problems (1.3) condition $\frac{\delta}{E} \in \sigma\left(K(t)^{p+1}\right)$ can never hold for sufficiently small $\delta$, hence this condition excludes the class of well-posed problems.

From (1.6) we realize that there exists no method $R(t): H \rightarrow H$ which guarantees an error bound $\left\|R(t) z^{\delta}-q(t)\right\|<E^{1-\frac{t}{T}} \delta^{\frac{t}{T}}$. On the other hand it is well known (cf. [11, $17,20]$ ) that there exist special regularization methods $R_{\alpha}(t)$ (where $R_{\alpha}(t): H \rightarrow H$ denotes a continuous operator depending on a positive regularization parameter $\alpha>0$ ) which guarantee the 'optimal' estimate

$$
\left\|R_{\alpha}(t) z^{\delta}-q(t)\right\| \leq E^{\frac{1}{1+p}} \delta^{\frac{p}{1+p}}=E^{1-\frac{t}{T}} \delta^{\frac{t}{T}}
$$

which means (together with (1.6)) that the best possible worst case error for identifying $q(t)$ from noisy data $z^{\delta} \in H$ under the conditions (A1), (A2) is given by

$$
\omega(\delta, t)=E^{1-\frac{t}{T}} \delta^{\frac{1}{T}} .
$$

We note that it has long been known that problem (1.3) can be stabilized by imposing condition (A2). By the concept of logarithmic convexity it can be shown (cf. [2, 14, 17]) that under the a priori assumption (A2) there holds $\|q(t)\| \leq E^{1-\frac{t}{T}}\|q(T)\|^{\frac{t}{T}}$ which means that $q(t)$ depends in a Hölder continuous way on the (unperturbed) final data $q(T)$. Thanks (1.6) and (1.7), (1.8) the following definition make sense, which is due to Vainikko et al. [21]: 
Deflnition 1.1. Parameter dependent regularization methods $R_{\alpha}(t)$ in which regularized solutions $q_{\alpha}^{\delta}(t)$ for problem (1.3) are defined according to $q_{\alpha}^{\delta}(t)=R_{\alpha}(t) z^{\delta}$ are called

(i) optimal on the set $M_{p, E}$ if $\Delta\left(\delta, R_{\alpha}(t)\right) \leq \omega(\delta, t)$ for a given parameter choice $\alpha=\alpha(\delta)$,

(ii) order optimal on the set $M_{p, E}$ if $\Delta\left(\delta, R_{\alpha}(t)\right) \leq c \omega(\delta, t)$ for a given parameter choice $\alpha=\alpha(\delta)$ where $c \geq 1$ is a constant independent of $\delta$.

For a discussion of optimality of parameter dependent regularization methods $R_{\alpha}^{\prime}(t)$ we refer to $[11,15,16,19,20]$; concerning order optimality we refer to $[1,2,11,21]$.

In this paper we are interested in optimality results for heat equation problems backward in time. We consider regularization methods of the general form

$$
q_{\alpha}^{\delta}(t)=R_{\alpha}(t) z^{\delta}=\sum_{i=1}^{\infty} \rho\left(\alpha, \kappa_{i}, t\right)\left(z^{\delta}, u_{i}\right) u_{i}
$$

and ask the question how (for given filter factors $\rho=\rho(\alpha, \kappa, t)$ ) to choose the regularization parameter $\alpha>0$ such that (1.9) becomes an optimal regularization method. A special optimal regularization method for backward heat equation problems (1.3) has been derived by Seidman [17]. This method is characterized by (1.9) with filter factors

$$
\rho=\min \left\{\frac{1}{\kappa_{i}(t)}, \frac{t}{T}\left(\frac{E}{\delta}\right)^{1-\frac{t}{T}}\right\}
$$

where $\kappa_{i}(t)=e^{-\lambda_{i}(T-t)}$ are the eigenvalues of $K(t) \in \mathcal{L}(H, H)$ (cf. also Remark 3.3).

\section{Optimality for a general regularization scheme}

From (1.9), (1.3) and (1.5) there follows

$$
\begin{aligned}
q(t)-q_{\alpha}^{\delta}(t) & =q(t)-R_{\alpha}(t) z+R_{\alpha}(t) z^{\prime}-R_{\alpha}(t) z^{\delta} \\
& =\left[I-R_{\alpha}(t) K(t)\right] K(t)^{p} q(0)+R_{\alpha}(t)\left[z-z^{\delta}\right]
\end{aligned}
$$

consequently we obtain from Lemma 2.2 in [19] (cf. also [12] and [4]) the following result.

Lemma 2.1. Let $q_{\alpha}^{\delta}(t)=R_{\alpha}(t) z^{\delta}$ the regularized solution (1.9). Then for the maximal error $\Delta\left(\delta, R_{\alpha}(t)\right)$ there holds.

$$
\Delta\left(\delta, R_{\alpha}(t)\right)=\inf _{0<\xi<1}\left\|\frac{E^{2}}{\xi} K(t)^{2 p}\left[I-R_{\alpha}(t) K(t)\right]^{2}+\frac{\delta^{2}}{1-\xi} R_{\alpha}^{2}(t)\right\|^{\frac{1}{2}}
$$

where $p$ is given by (1.5). 
In our following considerations we consider special regularization methods (1.9): For any fixed $t \in[0, T)$ we define an approximate solution $q_{\alpha}^{\delta}(t)$ for problem (1.3) according to

$$
q_{\alpha}^{\delta}(t)=g_{\alpha}\left(K(t)^{2 s+2}\right) K(t)^{2 s+1} z^{\delta}
$$

with $s \geq-\frac{1}{2}$. Here, $g_{\alpha}(\kappa):\left[0, \kappa_{1}\right] \rightarrow \mathbb{R}$ is a family of (piecewise) continuous functions depending on a positive regularization parameter $\alpha>0$ and $g_{\alpha}(K(t))$ is defined according to $g_{\alpha}(K(t)) q=\sum_{i=1}^{\infty} g_{\alpha}\left(\kappa_{i}\right)\left(q, u_{i}\right) u_{i}$ where $\kappa_{i}$ with $\kappa_{1} \geq \kappa_{2} \geq \ldots$ are the eigenvalues and $u_{i}$ the eigenelements of the linear compact operator $K(t) \in \mathcal{L}(H, H)$. Note that (2.3) has the general form (1.9) with

$$
\rho\left(\alpha, \kappa_{i}, t\right)=g_{\alpha}\left(\kappa_{i}^{2 s+2}\right) \kappa_{i}^{2 s+1} .
$$

Example 2.2 (Tikhonov regularization). This regularization method is characterized by the choice $g_{\alpha}(\kappa)=\frac{1}{\kappa+\alpha}$. The computation of the regularized solution (2.3) requires in this case to solve the linear operator equation

$$
\left(K(t)^{2 s+2}+\alpha I\right) q_{\alpha}^{\delta}=K(t)^{2 s+1} z^{\delta} .
$$

Example 2.3 (Regularized singular value decomposition). This regularization method is characterized by the choice

$$
g_{\alpha}(\kappa)= \begin{cases}\kappa^{-1} & \text { for } \kappa \geq \alpha \\ \alpha^{-\mu} \kappa^{\mu-1} & \text { for } \kappa<\alpha\end{cases}
$$

with some additional fixed parameter $\mu \in(0, \infty)$. The computation of the regularized solution (2.3) can be done by one of the following equivalent representations

$$
\begin{aligned}
q_{\alpha}^{\delta}(t) & =\sum_{\kappa_{i}^{2++2} \geq \alpha} \frac{\left(z^{\delta}, u_{i}\right)}{\kappa_{i}} u_{i}+\frac{1}{\alpha^{\mu}} \sum_{\kappa_{i}^{2 \alpha+2}<\alpha} \kappa_{i}^{\beta}\left(z^{\delta}, u_{i}\right) u_{i} \\
& =\sum_{\kappa_{i}^{2++2} \geq \alpha} \frac{\left(z^{\delta}, u_{i}\right)}{\kappa_{i}} u_{i}+\frac{1}{\alpha^{\mu}}\left\{K(t)^{\beta} z^{\delta}-\sum_{\kappa_{i}^{2 \alpha+2} \geq \alpha} \kappa_{i}^{\beta}\left(z^{\delta}, u_{i}\right) u_{i}\right\}
\end{aligned}
$$

with $\beta=2 \mu(s+1)-1$, where the second representation requires only the computation of a finite number of summands in the above sums.

Example 2.4 (Asymptotical regularization method). In this method the regularized solution $q_{\alpha}^{\delta}(t)$ is characterized by $(2.3)$ with $g_{\alpha}(\kappa)=\frac{1-e^{-\kappa / \alpha}}{\kappa}$, hence, there holds $q_{\alpha}^{\delta}(t)=$ $y\left(\frac{1}{\alpha}\right)$ where $y(\tau)$ is the solution of the initial value problem

$$
\left.\begin{array}{rl}
\vdots \dot{y}(\dot{\tau})+K(t)^{2 s+2} y(\tau) & =K(t)^{2 s+1} z^{6} \quad \text { for } \quad \tau \in\left(0, \frac{1}{\alpha}\right] \\
y(0) & =0
\end{array}\right\}
$$


- Example 2.5 (Iterated Tikhonov regularization). In this regularization method $q_{\alpha}^{\delta}(t)$ is obtained after performing $m$ steps of the method of Tikhonov regularization as discussed in Example 2.2, i.e. there holds $q_{\alpha}^{\delta}(t):=q_{m}$, where $q_{m}$ is obtained via

$$
\begin{aligned}
q_{0} & =0 \\
q_{k+1} & =q_{k}+\left(\dot{K}(t)^{2 s+2}+\alpha I\right)^{-1} K(t)^{2 s+1}\left(z^{\delta}-K(t) q_{k}\right) \quad(k=0(1)(m-1)) .
\end{aligned}
$$

Consequently, $q_{\alpha}^{\delta}(t)$ can be represented in the form (2.3) with $g_{\alpha}(\kappa)=\left[1-\left(\frac{\alpha}{\alpha+\kappa}\right)^{m}\right] / \kappa$.

Example 2.6 (Quasireversibility method). In this regularization method the regularized solution $q_{\alpha}^{\delta}(t)=u(t)$ is obtained by solving (backward in time) the regularized problem

$$
\left.\begin{array}{rl}
u_{\tau}+A u(\tau)-\alpha A^{2} u(\tau) & =0 \quad \text { for } t \leq \tau<T \\
u \quad u(T) & =z^{\delta}
\end{array}\right\}
$$

Since $u(\tau)(\tau \in[t, T))$ can be represented by

$$
\begin{aligned}
u(\tau) & =\sum_{i=1}^{\infty} e^{\left(-\alpha \lambda_{i}^{2}+\lambda_{i}\right)(T-\tau)}\left(z^{\delta}, u_{i}\right) u_{i} \\
& =\sum_{i=1}^{\infty} \kappa_{i}^{-1-\frac{\alpha \ln \alpha_{i}}{T-\tau}}\left(z^{\delta}, u_{i}\right) u_{i}
\end{aligned}
$$

we find that the regularized solution $q_{\alpha}^{\delta}(t)$ can be represented in the form (1.9) with $\rho\left(\alpha, \kappa_{i}, t\right)=\kappa_{i}^{-1-\frac{\alpha \ln \kappa_{i}}{T-i}}$, or equivalently, in the form (2.3) where in case $s=-\frac{1}{2}$ there holds $g_{\alpha}(\kappa)=\kappa^{-1-\frac{\alpha \ln \kappa}{T-t}}$.

Now we introduce for $0 \leq \kappa<\infty$ and $0<\alpha<\infty$ the functions $g:[0, \infty) \rightarrow \mathbb{R}$ and $h:[0, \infty) \rightarrow \mathbb{R}$ according to

$$
g(\kappa)=\alpha g_{\alpha}(\alpha \kappa) \quad \text { and } \quad h(\kappa)=1-\kappa g(\kappa)
$$

and assume that $g$ and $h$ are independent of the regularization parameter $\alpha$. Note that (2.11) is satisfied for Examples $2.2-2.5$ but violated for Example 2.6. We easily find that $g(\kappa)=\frac{1}{\kappa+1}$ for Example 2.2,

$$
g(\kappa)= \begin{cases}\kappa^{-1} & \text { for } \kappa \geq 1 \\ \kappa^{\mu-1} & \text { for } \kappa<1\end{cases}
$$

for Example 2.3, $g(\kappa)=\frac{1-e^{-\kappa}}{\kappa}$ for Example 2.4 and $g(\kappa)=\left[1-\left(\frac{1}{1+\kappa}\right)^{m}\right] / \kappa$ for Example 2.5 .

For regularization methods (2.3) satisfying (2.11) we obtain from Lemma 2.1 in analogy to the proof of Theorem 3.1 in [20] (cf. also Theorem 2.3 in [15]) the following result. 
Theorem 2.7. Let $g$ and $h$ the functions defined by (2.11) and $p$ the parameter given by (1.5). If

(i) the equation $h(\kappa)=\frac{1}{1+p}$ has a unique solution $\kappa_{0}$ and

(ii) $\alpha$ is chosen by $\alpha=\frac{1}{\kappa_{0}}\left(\frac{\delta}{E}\right)^{\frac{2++2}{p+1}}$,

then for $q_{\alpha}^{\delta}(t)=R_{\alpha}(t) z^{\delta}$ defined in (2.3) there holds the error estimate

$$
\Delta\left(\delta, R_{\alpha}(t)\right) \leq E^{\frac{1}{1+p}} \delta^{\frac{p}{1+p}} \sup _{0 \leq \kappa<\infty}\{f(\kappa)\}^{\frac{1}{2}}=E^{1-\frac{l}{T}} \delta^{\frac{2}{T}} \sup _{0 \leq \kappa<\infty}\{f(\kappa)\}^{\frac{1}{2}}
$$

with

$$
f(\kappa)=(1+p)\left(\frac{\kappa}{\kappa_{0}}\right)^{\frac{p}{0+1}} h^{2}(\kappa)+\frac{1+p}{p} \cdot\left(\frac{\kappa}{\kappa_{0}}\right)^{-\frac{1}{1+0}} \kappa^{2} g^{2}(\kappa):
$$

Remark 2.8. Theorem 2.7 shows that regularization methods (2.3) satisfying (2.11) are optimal if $\sup _{\kappa \in[0, \infty)} f(\kappa) \leq 1$. If $f(\kappa)>1$ for some $\kappa \in[0, \infty)$ and $[0, \varepsilon] \subset \sigma(K(t))(\varepsilon>0)$, then method (2.3) is not optimal for any parameter choice $\alpha=\alpha(\delta)$ provided $\delta=\delta(\varepsilon)$ is sufficiently small. For the proof of some non-optimality results we refer to $[11,19,20]$.

\section{Optimality for special regularization methods}

On the basis of Theorem 2.7 it is possible to obtain optimality results for different regularization.methods discussed in Examples $2.2-2.5$. Let us start to consider the method of Tikhonov regularization (Example 2.2 with $s \doteq 0$ ). In this method the functions $g$ and $h$ from (2.11) are given by $g(\kappa)=\frac{1}{\kappa+1}$ and $h(\kappa)=\frac{1}{\kappa+1}$. The equation $h(\kappa)=\frac{1}{p+1}$ has the unique solution $\kappa_{0}=p$, hence, the function $f$ from (2.14) attains the form

$$
f(\kappa)=\frac{(1+p) \kappa^{\dot{p}}}{p^{p}(\kappa+1)^{2}}+\frac{(1+p) \kappa}{(\kappa+1)^{2}} .
$$

Since supp $\sin _{\kappa} f(\infty) \leq 1$ for all $p \in[0,2]$ we obtain from Theorem 2.7 and (1.5) the well known result that for the a priori parameter choice $\alpha=\frac{1}{p}\left(\frac{\delta}{E}\right)^{\frac{2}{p+1}}$ the method of Tikhonov regularization (Example 2.2 with $s=0$ ) is optimal for $p \in[0,2]$, hence there holds

Theorem 3.1. Let, for any fixed $t \in[0, T), q_{\alpha}^{\delta}(t)=R_{\alpha}(t) z^{\delta}$ the regularized solu: tion (2.5) with $s=0$ where $\alpha$ is chosen by $\alpha=\frac{T-t}{t}\left(\frac{\delta}{E}\right)^{2 \frac{T-t}{T}}$. If $t \in\left[0, \frac{2}{3} T\right]$, then the error estimate

$$
\Delta\left(\delta, R_{\alpha}(t)\right) \leq E^{1-\frac{1}{T}} \delta^{\frac{i}{T}}
$$

holds.

In analogy to Theorem 3.1 it is possible to derive optimality results for further regularization methods. Some of the results which can be deduced from Theorem 2.7 can be formulated as follows: 
(i) The method of Tikhonov regularization with $s=-\frac{1}{2}$ (cf. Example 2.2) is optimal for any fixed $t \in\left[0, \frac{3-\sqrt{5}}{2} T\right]$ if $\alpha$ is chosen by $\alpha=\frac{T-t}{t}\left(\frac{\delta}{E}\right)^{\frac{T-t}{T}}$.

(ii) The method of asymptotical regularization with $s=0$ (cf. Example 2.4) is optimal for $p \in[0,7.12]$, i.e. for all $t \in[0,0.88 T]$ if $\alpha$ is chosen by $\alpha=\left(\frac{\delta}{E}\right)^{2 \frac{T-t}{T}} /\left[\ln \frac{T}{T-t}\right]$.

(iii) The method of iterated Tikhonov regularization with $s=0$ and $m=2$ (cf. Example 2.5) is optimal for $p \in[0,3.58]$, i.e. for all $t \in[0,0.78 T]$ if $\alpha$ is chosen by $\alpha=\frac{\sqrt{T-t}}{\sqrt{T}-\sqrt{T-1}}\left(\frac{\delta}{E}\right)^{2 \frac{T-t}{T}}$.

The following Table 1 contains the optimal values $\alpha_{\text {opt }}$ of the regularization parameter and the corresponding $\frac{t}{T}$-ranges for which the methods of Tikhonov regularization (Example 2.2), iterated Tikhonov regularization with $m=2$ (Example 2.4) and the method of asymptotical regularization (Example 2.5) are optimal.

\begin{tabular}{|c|c|c|c|}
\hline $\begin{array}{c}\text { Regularization } \\
\text { method }\end{array}$ & $\begin{array}{c}\text { Tikhonov } \\
\text { regularization }\end{array}$ & $\begin{array}{c}\text { iterated Tikhonov } \\
\text { regularization with } m=2\end{array}$ & $\begin{array}{c}\text { asymptotical } \\
\text { regularization }\end{array}$ \\
\hline$\alpha_{\text {opt }}$ & $\frac{T-t}{t}\left(\frac{\delta}{E}\right)^{\nu}$ & $\frac{\sqrt{T-t}}{\sqrt{T}-\sqrt{T-t}}\left(\frac{\delta}{E}\right)^{\nu}$ & {$\left[\ln \frac{T}{T-t}\right]^{-1}\left(\frac{\delta}{E}\right)^{\nu}$} \\
\hline$s=-0.5$ & $0: 0.382$ & $0: 0.434$ & $0: 0.511$ \\
$s=0.0$ & $0: 0.667$ & $0: 0.782$ & $0: 0.877$ \\
$s=0.5$ & $0.051: 0.750$ & $0.078: 0.857$ & $0.146: 0.932$ \\
$s=1.0$ & $0.196: 0.800$ & $0.265: 0.889$ & $0.399: 0.955$ \\
$s=1.5$ & $0.329: 0.833$ & $0.416: 0.909$ & $0.564: 0.966$ \\
$s=2.0$ & $0.432: 0.857$ & $0.525: 0.923$ & $0.669: 0.974$ \\
\hline
\end{tabular}

Table 1. $\frac{t}{T}$-ranges of optimality, $\nu:=2(s+1) \frac{T-t}{T}$

Note that outside of the $\frac{t}{T}$-ranges given in Table 1 the corresponding regularization methods are not optimal, hence, there appear saturation effects. Such saturation effects have already been known in case of order optimality (cf. [5]). For example, Tikhonov regularization with $s=0$ is order optimal for the parameter choice $\alpha \sim \delta^{2\left(1-\frac{t}{T}\right.}$ provided $\frac{t}{T} \in\left[0, \frac{2}{3}\right]$; for $\frac{t}{T}>\frac{2}{3}$ Tikhonov regularization with $s=0$ is not order optimal.

In our following considerations we discuss the method of regularized singular value decomposition (cf. Example 2.3). The next theorem shows that for this method optimality can be guaranteed for arbitrary values $\frac{t}{T} \in[0,1)$, hence, there doesn't appear any saturation effect.

Theorem 3.2. Let, for any fixed $t \in[0, T), q_{\alpha}^{\delta}(t)=R_{\alpha}(t) z^{\delta}$ the regularized solution (2.6) where $\alpha$ is given by

$$
\alpha=\left(\frac{T}{t}\right)^{\frac{1}{\mu}}\left(\frac{\delta}{E}\right)^{\frac{2(\imath+1)(T-1)}{T}} .
$$

If the constants $\mu$ and $s$ are related by $1 \leq 2 \mu(s+1) \leq 2$, then for any $t \in[0, T)$ the optimal error estimate (3.2) holds. 
Proof. We apply Theorem 2.7 with function $g$ given by (2.12) and prove that $\sup _{\kappa \geq 0} f(\kappa)<1$ if $\mu \leq \frac{1}{1+s} \leq 2 \mu$ holds, where $f(\kappa)$ is given by (2.14). Let $h$ the function defined by (2.11). Then, since the equation $h(\kappa)=\frac{1}{p+1}$ with $p$ given by (1.5) has the unique solution $\kappa_{0}=\left(\frac{p}{p+1}\right)^{\frac{1}{\mu}}$ we obtain that $f(\kappa)$ is given by

$$
\begin{aligned}
& f(\kappa)= \\
& \begin{cases}\left(\frac{1+p}{p}\right)^{1-\frac{1}{\mu(0+1)}} \kappa^{-\frac{1}{\rho+1}} & \text { for } \kappa \geq 1 \\
(1+p)\left(\frac{1+p}{p}\right)^{\frac{p}{p(0+1)}} \kappa^{\frac{p}{0+1}}\left(1-\kappa^{\mu}\right)^{2}+\left(\frac{1+p}{p}\right)^{1-\frac{1}{\mu(\rho+1)}} \kappa^{2 \mu-\frac{1}{\rho^{\prime}+1}} & \text { for } \kappa<1\end{cases}
\end{aligned}
$$

First we note that $\sup _{\kappa \geq 0} f(\kappa)<\infty$ if $0<\frac{1}{s+1} \leq 2 \mu$ holds. Let $0<\frac{1}{s+1} \leq 2 \mu$. Then $f(\kappa)$ is monoton decreasing for $\kappa \geq 1$. Furthermore, $f(1) \leq 1$ is satisfied for $\mu \leq \frac{1}{s+1}$. Hence it remains to prove that for $\mu \leq \frac{1}{s+1} \leq 2 \mu$ there holds sup $_{0 \leq \kappa \leq 1} f(\kappa) \leq 1$. For $0 \leq y \leq 1$ there holds $y^{p}(1-y) \leq\left[\frac{p}{1+p}\right]^{p}\left[\frac{1}{1+p}\right]$, consequently, for $0 \leq \kappa^{\mu} \leq 1$ there holds the inequality

$$
\left(\kappa^{\mu}\right)^{\frac{p}{\rho+1}}\left(1-\kappa^{\mu}\right)^{\frac{1}{\rho+1}} \leq\left(\frac{p}{1+p}\right)^{\frac{p}{\rho+1}}\left(\frac{1}{1+p}\right)^{\frac{1}{\rho+1}}
$$

which gives

$$
\kappa^{\frac{p}{0+1}} \leq\left(\frac{p}{1+p}\right)^{\frac{p}{\mu(0+1)}}\left(\frac{1}{p+1}\right)^{\frac{1}{\mu(0+1)}}\left(1-\kappa^{\mu}\right)^{-\frac{1}{\mu(0+1)}} .
$$

We use this inequality and obtain from (3.4)

$$
f(\kappa) \leq(1+p)^{1-\frac{1}{\mu(\alpha+1)}}\left(1-\kappa^{\mu}\right)^{2-\frac{1}{\mu(0+1)}}+\left(\frac{p}{1+p}\right)^{\frac{1}{\mu(0+1)}-1}\left(\kappa^{\mu}\right)^{2-\frac{1}{\mu(\cdot+1)}}
$$

for $0 \leq \kappa^{\mu} \leq 1$. One shows that the right-hand side of this inequality attains its maximum for $\kappa^{\mu} \cdot=\frac{p}{1+p}$ which yields the result $\sup _{0 \leq \kappa \leq 1} f(\kappa) \leq f\left(\left(\frac{p}{1+p}\right)^{\frac{1}{\mu}}\right)=1$

Remark 3.3. Recommendations for the choice of $s$ and $\mu$ in Theorem 3.2 are values with $2 \mu(s+1)=1$ (e.g. $s=-\frac{1}{2}$ and $\mu=1$ ) since in this case the number of summands to be computed in the finite sums of (2.6) is 'small'. In this case we obtain immediately from Theorem 3.2 that for the regularized solution

$$
q_{\alpha}^{\delta}(t)=\sum_{\kappa_{i} \geq \alpha} \frac{\left(z^{\delta}, u_{i}\right)}{\kappa_{i}} u_{i}+\frac{1}{\alpha}\left\{z^{\delta}-\sum_{\kappa_{i} \geq \alpha}\left(z^{\delta}, u_{i}\right) u_{i}\right\}
$$

with

$$
\kappa_{i}=\kappa_{i}(t)=e^{-\lambda_{i}(T-t)} \quad \text { and } \quad \alpha=\alpha(t)=\frac{T}{t}\left(\frac{\delta}{E}\right)^{\frac{T-t}{T}}
$$

there holds (for any fixed $t \in[0, T)$ ) the optimal error estimate (3.2). Note that the representation (3.5) is equivalent to the representation (1.9), (1.10) from the paper of Seidman (cf. [17]). Furthermore note that for smaller $t$-values a smaller number of summands in the finite sums of (3.5) is needed. 


\section{Optimality for quasireversibility methods}

If the eigenvalues and eigenelements of the operator $A$ of equation (1.1) are available, then the method of regularized singular value decomposition as proposed in Example 2.3 is definitely to be recommended since it appears to be

(a) very cheap concerning the computational amount of work if one decides to use the representation (3.5) and

(b) optimal for any fixed $t \in[0, T$ ) (cf. Theorem 3.2 and Remark 3.3).

However only in very special circumstances - constant coefficients and simple geometries for example - it will be possible to compute the eigenvalues and eigenelements of a partial differential operator $\mathrm{A}$ analytically. Generally the eigenvalues and eigenelements of $A$ will not be available. In this case the method of regularized singular value decomposition becomes very expensive concerning the computational amount of work.

The methods discussed in Examples 2.2, 2.4 and 2.5 do not require the knowledge of the eigenvalues and eigenelements of the partial differential operator $A$, but they have the disadvantage that they are not optimal for all $t \in[0, T)$ and they have the difficulty that

(i) the generation of the (discretized) operator $K(t)$ is quite expensive and

(ii) the (discretized) operator $K(t)$ is dense, even if $A$ is sparse.

Of course, these disadvantages can be overcome if one switches over to the concept of adjoint equations from control theory which does not require to generate $K(t)$. It is clear that, e.g. for integer values of $2 s+1$, the element $K(t)^{2 s+1} z^{\delta}$ can be computed without to generate $K(t)$ explicitely. Also for the realization of (2.5) it is possible to use iteration methods which do not require to generate $K(t)$ explicitely.

We note a further disadvantage of the methods that fit into the framework of Theorem 2.7. This disadvantage appears if the solution $q(t)$ is searched not only for one fixed $t \in[0, T)$ but for all $t \in[0, T)$. Then the considered methods have to be applied for a series of $t$-values $0=t_{0}<t_{1}<\ldots<t_{n}<T$ and it is not clear how to use the information of $q_{\alpha}^{\delta}\left(t_{i}\right)$ in order to compute an 'optimal' regularized approximation $q_{\alpha}^{\delta}\left(t_{i-1}\right)$.

A very cheap method from the point of computational amount is the method of quasireversibility proposed in [10] (cf. Example 2.6). A generalization of this method can be described as follows: search for $q_{\alpha}^{\delta}(t)=u(t)$ by solving (backward in time) the regularized problem

$$
\left.\begin{array}{rl}
u_{\tau}(\tau)+g_{\alpha}(A) u(\tau) & =0 \quad \text { for } \quad t \leq \tau<T \\
u(T) & =z^{\delta}
\end{array}\right\}
$$

with a function $g_{\alpha}$ to be chosen appropriately. Note that $g_{\alpha}(\lambda)=\lambda-\alpha \lambda^{2}$ for Example 2.6. Since for any fixed $t \in[0, T)$ the solution $q_{\alpha}^{\delta}(t)=u(t)$ of $(4.1)$ can be represented by

$$
q_{\alpha}^{\delta}(t)=\sum_{i=1}^{\infty} e^{g_{\alpha}\left(\lambda_{i}\right)(T-t)}\left(z^{\delta}, u_{i}\right) u_{i}
$$


we find that $q_{\alpha}^{\delta}(t)$ can be represented in the form (1.9) or (2.3), respectively, but (2.11) will generally be violated such that Theorem 2.7 cannot be applied in order to find optimality results for method (4.2).

In our following considerations we ask the question if it is possible to find some optimality results for method (4.1). Since Theorem 2.7 cannot be applied we start our examinations by applying Lemma 2.1 and obtain the following

Lemma 4.1. Let, for any fixed $t \in[0, T), q_{\alpha}^{\delta}(t)=R_{\alpha}(t) z^{\delta}$ defined by (4.2). Then for the maximal error $\Delta\left(\delta, R_{\alpha}(t)\right)$ of $(1.8)$ there holds

$$
\begin{aligned}
& \Delta\left(\delta, R_{\alpha}(t)\right)= \\
& \inf _{\xi \in(0,1)} \sup _{\lambda \in\left[\lambda_{1}, \infty\right)}\left\{\frac{E^{2}}{\xi} e^{-2 \lambda p(T-t)}\left[1-e^{\left(g_{\alpha}(\lambda)-\lambda\right)(T-t)}\right]^{2}+\frac{\delta^{2}}{1-\xi} e^{2 g_{\alpha}(\lambda)(T-t)}\right\}^{\frac{1}{2}}
\end{aligned}
$$

where $p$ is given by (1.5).

In order to derive optimality results in an unified manner we introduce the two functions $g:[0, \infty) \rightarrow \mathbb{R}$ and $h:[0, \infty) \rightarrow \mathbb{R}$ according to

$$
g(\lambda)=\alpha g_{\alpha}\left(\frac{\lambda}{\alpha}\right) \quad \text { and } \quad h(\lambda)=1-\frac{g(\lambda)}{\lambda}
$$

and assume that $g$ and $h$ are independent of the regularization parameter $\alpha$. Let us discuss some examples.

Example 4.2 (Quasireversibility method of Lattès and Lions, cf. [10]). In this regularization method the regularized solution $q_{\alpha}^{\delta}(t)=u(t)$ is obtained by solving (backward in time) the regularized problem (4.1) with $g_{\alpha}(A)=A-\alpha A^{2}$. Consequently, this method is characterized by (4.2) with $g_{\alpha}(\lambda)=\lambda-\alpha \lambda^{2}$, hence, (4.4) is satisfied with $g(\lambda)=\lambda-\lambda^{2}$ and $h(\lambda)=\lambda$.

Example 4.3 (Quasireversibility method of Gajewski and Zacharias, cf. [3]). In this regularization method the regularized solution $q_{\alpha}^{\delta}(t)=u(t)$ is obtained by solving (backward in time) the regularized problem

$$
\left.\begin{array}{rl}
\frac{\partial}{\partial \tau}[u(\tau)+\alpha A u(\tau)]+A u(\tau) & =0 \quad \text { for } \quad t \leq \tau<T \\
u(T) & =z^{\delta}
\end{array}\right\} .
$$

Since the regularized solution $q_{\alpha}^{\delta}(t)=u(t)$ of problem (4.5) can be represented by

$$
q_{\alpha}^{\delta}(t)=\sum_{i=1}^{\infty} e^{\frac{\lambda_{i}\left(T-t^{2}\right)}{1+\alpha \lambda_{i}}}\left(z^{\delta}, u_{i}\right) u_{i}
$$

it follows that $q_{\alpha}^{\delta}(t)$ has the representation (4.2) with $g_{\alpha}(\lambda)=\frac{\lambda}{1+\alpha \lambda}$. Consequently (4.4) holds with $g(\lambda)=\frac{\lambda}{1+\lambda}$ and $h(\lambda)=\frac{\lambda}{1+\lambda}$. 
Example 4.4 (Quasireversibility method of degree $n$, cf. [13]). In this regularization method the regularized solution $q_{\alpha}^{\delta}(t)=u(t)$ is obtained by solving (backward in time) the regularized problem

$$
\left.\begin{array}{rl}
u_{\tau}(\tau)+A u(\tau)-\alpha^{n-1} A^{n} u(\tau) & =0 \quad \text { for } \quad t \leq \tau<T \\
u(T) & =z^{\delta}
\end{array}\right\}
$$

where $n \geq 2$ is an integer (note that $n=2$ corresponds with Example 4.2). It can be shown that the regularized solution $q_{\alpha}^{\delta}(t)=u(t)$ of problem (4.7) can be represented by (4.2) with $g_{\alpha}(\lambda)=\lambda-\alpha^{n-1} \lambda^{n}$, hence, (4.4) is satisfied with $g(\lambda)=\lambda-\lambda^{n}$. and $h(\lambda)=\lambda^{n-1}$.

For regularization methods (4.2) satisfying (4.4) we obtain from Lemma 4.1 with the substitution $y=\alpha \lambda$ that

$$
\left\ulcorner\Delta\left(\delta, R_{\alpha}(t)\right) \leq E^{\frac{1}{p+1}} \delta^{\frac{p}{p+1}} \inf _{\xi \in(0,1)} \sup _{y \in(0, \infty)}\{f(\xi, y, \alpha)\}^{\frac{1}{2}}\right.
$$

with

$$
\begin{aligned}
& f(\xi, y, \alpha)= \\
& \frac{1}{\xi}\left(\frac{\delta}{E}\right)^{-\frac{2 p}{p+1}} e^{-\frac{2}{\alpha} y p(T-t)}\left[1-e^{\frac{1}{\alpha}(g(y)-y)(T-t)}\right]^{2}+\frac{1}{1-\xi}\left(\frac{\delta}{E}\right)^{\frac{2}{p+1}} e^{\frac{2}{\alpha} g(y)(T-t)}
\end{aligned}
$$

From (4.8) we realize that method (4.2) is optimal provided

$$
\inf _{0<\xi<1} \sup _{0 \leq y<\infty} f(\xi, y, \alpha) \leq 1 .
$$

holds for a special choice of the regularization parameter. In order to determine an optimal regularization parameter we search for a stationary point of the function $f(\xi, y, \alpha)$.

Lemma 4.5. Let $g(y):[0, \infty) \rightarrow \mathbb{R}$ differentiable and $p$ given by (1.5). If the equation

$$
h(y)=(p+1) \frac{\ln \frac{p+1}{p}}{\ln \frac{E}{\delta}}
$$

has a solution $y_{0}$, then

$$
\left(\xi_{0}, y_{0}, \alpha_{0}\right)=\left(\frac{1}{p+1}, y_{0}, \frac{y_{0}(T-t)(p+1)}{\ln \frac{E}{\delta}}\right)
$$

is a stationary point of the function $f(\xi, y, \alpha)$. Furthermore there holds

$$
f\left(\xi_{0}, y_{0}, \alpha_{0}\right)=1
$$


Proof. One shows that the three equations $f_{\xi}^{\prime}=0, f_{y}^{\prime}=0$ and $f_{\alpha}^{\prime}=0$ are equivalent to the nonlinear system

$$
\begin{aligned}
\left(\frac{\delta}{E}\right)^{2} X^{2} e^{\frac{2}{a}(p+1) y(T-t)} & =\left(\frac{1-\xi}{\xi}\right)^{2}(1-X)^{2} \\
p(1-X)+\left[g^{\prime}(y)-1\right] X & =\frac{1-\xi}{\xi} g^{\prime}(y)(1-X) \\
y p(1-X)+[g(y)-y] X & =\frac{1-\xi}{\xi} g(y)(1-X)
\end{aligned}
$$

where $X$ stands shortly for $X=e^{(g(y)-y) \frac{T-1}{\alpha}}$. We realize that the last two equations are satisfied for the choice

$$
\xi=\frac{1}{1+p} \quad \text { and } \quad X=\frac{p}{1+p}
$$

Consequently it remains to solve the two equations

$$
\frac{\delta}{E}=e^{-\frac{1}{\alpha}(p+1) y(T-t)} \quad \text { and } \quad e^{-\frac{1}{a} h(y) y(T-t)}=\frac{p}{p+1}
$$

where $h$ is given by (4.4). From (4.13) and (4.14) we obtain that (4.11) is a stationary point of the function (4.9) where $y_{0}$ is the solution of the equation (4.10). We substitute (4.11) into (4.9) and obtain that (4.12) holds

Now we substitute $\xi_{0}$ and $\alpha_{0}$ into (4.9) and obtain together with (1.5) the following result.

Theorem 4.6. Let (4.4), hold, $g(y):(0, \infty) \rightarrow \mathbb{R}$ differentiable and $p$ given by (1.5). If

(i) the equation $h(y)=(p+1) \ln \frac{p+1}{p} / \ln \frac{E}{\delta}$ has a solution $y_{0} \cdot$ and

(ii) $\alpha$ is chosen by $\alpha=y_{0}(T-t)(p+1) / \ln \frac{E}{\delta}$,

then for $q_{\alpha}^{\delta}(t)=R_{\alpha}(t) z^{\delta}$ defined by (4.2) there holds the error estimate

$$
\Delta\left(\delta, R_{\alpha}(t)\right) \leq E^{\frac{1}{1+p}} \delta^{\frac{p}{1+p}} \sup _{0 \leq y<\infty}\{f(y)\}^{\frac{1}{2}}
$$

with

$$
\begin{aligned}
& f(y)= \\
& (p+1)\left(\frac{\delta}{E}\right)^{\frac{2 p}{p+1}\left(\frac{y}{y_{0}}-1\right)}\left[1-\left(\frac{\delta}{E}\right)^{\frac{h(y)}{p+1} \frac{1}{y_{0}}}\right]^{2}+\frac{p+1}{p}\left(\frac{\delta}{E}\right)^{\frac{2}{p+1}\left[1-\frac{1}{y_{0}}+\frac{1}{y_{0}} h(y)\right]}
\end{aligned}
$$


Remark 4.7. Theorem 4.6 shows that regularization methods (4.2) satisfying (4.4) are optimal if $\sup _{0 \leq y<\infty} f(y) \leq 1$ where $f(y)$ is given by (4.16); this in turn depends on the ratios $\frac{t}{T}$ and $\frac{\delta}{E}$. An analytical examination of the function $f$ shows that following properties are valid:

(i) $f\left(y_{0}\right)=1$ where $y_{0}$ is the solution of the equation (i) in Theorem 4.6.

(ii) $f^{\prime}\left(y_{0}\right)=0$.

(iii) $f^{\prime \prime}\left(y_{0}\right)=\frac{2 p}{(1+p)^{2}}\left(\frac{\ln \delta / E}{y_{0}}\right)^{2}\left[x^{2}-4 x+2\right]$ where $x=h\left(y_{0}\right)+y_{0} h^{\prime}\left(y_{0}\right), p$ is given by (1.5) and $h(y)$ is given by (4.4).

(iv) $f^{\prime \prime}\left(y_{0}\right)<0$ if and only if

$$
2-\sqrt{2}<h\left(y_{0}\right)+y_{0} h^{\prime}\left(y_{0}\right)<2+\sqrt{2} .
$$

Unfortunately we cannot conclude from (i) - (iv) that for $\frac{t}{T}$-values satisfying (4.17) the function $f$ in (4.16) has a global maximum at $y=y_{0}$. In order to check for which $\frac{t}{T}$-values $y_{0}$ is a global maximum of (4.16) (i.e. for which $\frac{t}{T}$-values quasireversibility methods (4.2) are optimal) it seems to be necessary to examine $f(y)$ numerically. On the other hand, (4.17) gives us a hint in which range optimality can be expected since we know that outside of the $\left(\frac{t}{T}, \frac{\delta}{E}\right)$-range defined by (4.17) the corresponding quasireversibility methods (4.2) are not optimal.

\section{Optimality for special quasireversibility methods}

In this section we consider the special quasireversibility methods discussed in Examples $4.2-4.4$ and ask the question for which values of $\frac{\delta}{E}$ and $\frac{t}{T}$ the corresponding methods are optimal provided the regularization parameter $\alpha$ has been chosen properly. In order to answer this question we will apply Theorem 4.6. Let us start to discuss the methods described in Examples 4.2 and 4.4. In these regularization methods the regularized solution $q_{\alpha}^{\delta}(t)$ is obtained from (4.7) with integer values $n \geq 2$. Consequently, for the functions $g$ and $h$ of (4.4) we have $g(\lambda)=\lambda-\lambda^{n}$ and $h(\lambda)=\lambda^{n-1}$. We suppose that $\delta<E$ holds, then we find that the equation $h(y)=(p+1) \ln \frac{p+1}{p} / \ln \frac{E}{\delta}$ (compare Theorem 4.6) has the unique positive solution

$$
y_{0}=\left\{(p+1) \frac{\ln \frac{p+1}{p}}{\ln \frac{E}{\delta}}\right\}^{\frac{1}{n-1}} .
$$

Hence, the regularization parameter $\alpha$ given in Theorem 4.6 (which leads to optimal error bounds (3.2)) has to be chosen optimally according to

$$
\alpha=\frac{T}{\ln \frac{E}{\delta}}\left\{\frac{T \ln \frac{T}{t}}{(T-t) \ln \frac{E}{\delta}}\right\}^{\frac{1}{n-1}}
$$

where we have used that $p$ is given by $p=\frac{t}{T-t}$. In order to apply Theorem 4.6 we have to examine the function $f$ given by (4.16). From Remark 4.7 we know that $f^{\prime \prime}\left(y_{0}\right)<0$ if and only if (4.17) holds. It can be shown that (4.17) is equivalent to the inequality 


$$
\left(\frac{t}{T}\right)^{\frac{n}{2} \frac{2+\sqrt{2}}{1-1 / T}}<\frac{\delta}{E}<\left(\frac{t}{T}\right)^{\frac{n}{2} \frac{2-\sqrt{2}}{1-t / T}} .
$$

Since outside of the $\left(\frac{t}{T}, \frac{\delta}{E}\right)$-range defined by (5.3) the corresponding quasireversibility methods (4.7) are not optimal it follows that $f(y)$ has only to be examined numerically inside the range defined by (5.3). In order to check for which $\left(\frac{t}{T}, \frac{\delta}{E}\right)$-values $y_{0}$ is a global maximum of (4.16) we have executed numerical experiments (for different integer values $n \geq 2$ ). We have found that for $\left(\frac{t}{T}, \frac{\delta}{E}\right)$-values that belong to the ranges given in Figures $1-3$ there holds $\sup _{y \geq 0} f(y) \leq 1$. Note that the ranges defined by (5.3) are something larger than the ranges of Figures $1-3$ for which the corresponding regularization methods (4.7) are optimal. Summing up we have found the following results.

Theorem 5.1. Let $q_{\alpha}^{\delta}(t)=R_{\alpha}(t) z^{\delta}$ the regularized solution defined in Example 4.4 with $n=2, n=3$ or $n=4$, respectively, where the regularization parameter $\alpha$ is chosen according to (5.2). If $\left(\frac{t}{T}, \frac{\delta}{E}\right)$ belongs to the range given in Figure 1, Figure 2 or Figure 3 , respectively, then there holds the optimal error estimate (3.2).

In the second part of this section we are going to discuss optimality results for the quasireversibility method of Gajewski and Zacharias (cf. Example 4.3). In this method the regularized solution $q_{\alpha}^{\delta}(t)$ can be represented by (4.6), hence, for the functions $g$ and $h$ of (4.4) we have $g(\lambda)=h(\lambda)=\frac{\lambda}{1+\lambda}$. We suppose that

$$
\frac{\delta}{E}<\left(\frac{t}{T}\right)^{\frac{1}{i-t / T}}
$$

holds. Then we find that the equation $h(y)=(p+1) \ln \frac{p+1}{p} / \ln \frac{E}{\delta}$ (compare Theorem 4.6) has the unique positive solution

$$
y_{0}=\frac{(p+1) \ln \frac{p+1}{p}}{\ln \frac{E}{\delta}-(p+1) \ln \frac{p+1}{p}} .
$$

Hence, the (positive) regularization parameter $\alpha$ given in Theorem 4.6 (which leads to optimal error bounds (3.2)) has to be chosen according to

$$
\alpha=\frac{T^{2} \ln \frac{T}{t}}{\ln \frac{E}{\delta}\left\{(T-t) \ln \frac{E}{\delta}-T \cdot \ln \frac{T}{8}\right\}}
$$

The numerical computed $\left(\frac{t}{T}, \frac{\delta}{E}\right)$-range for which $\sup _{y \geq 0} f(y) \leq 1$ holds is given in Figure 4. Since all points of this range satisfy the inequality (5.4) there holds the following

Theorem 5.2. Let $q_{\alpha}^{\delta}(t)=R_{\alpha}(t) z^{\dot{\delta}}$ the regularized solution defined in Example 4.3 where the regularization parameter $\alpha$ is chosen according to (5.5). If $\left(\frac{t}{T}, \frac{\delta}{E}\right)$ belongs to the-range given in Figure 4, then there holds the optimal error estimate (3.2). 
Figure 1: $\left(\frac{t}{T}, \frac{\delta}{E}\right)$-range $B$ of optimality for method (4.7) with $n=2$
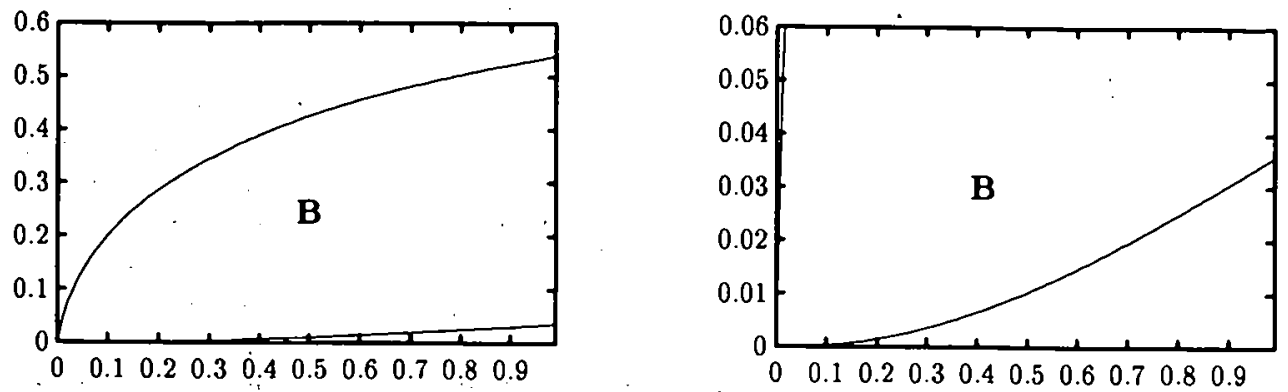

Figure 2: $\left(\frac{t}{T}, \frac{\delta}{E}\right)$-range $B$ of optimality for method (4.7) with $n=3$
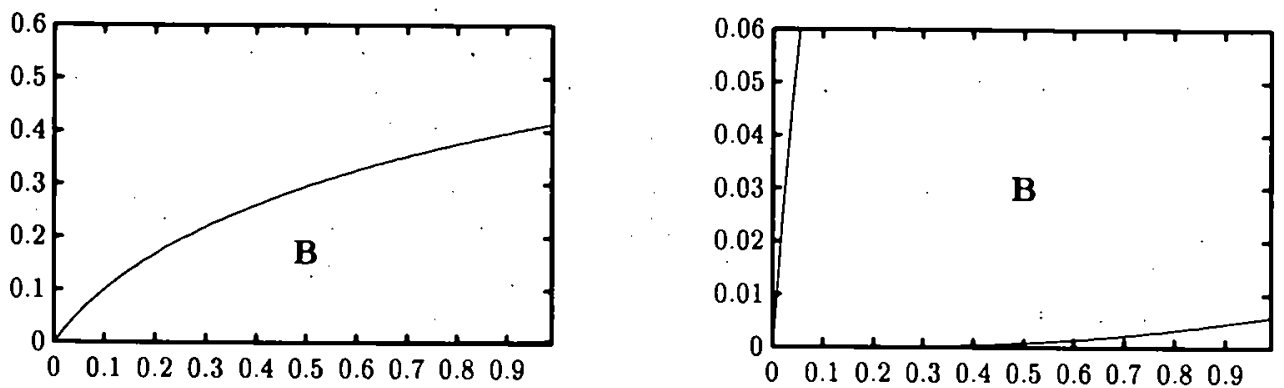

Figure 3: $\left(\frac{t}{T}, \frac{\delta}{E}\right)$-range $B$ of optimality for method (4.7) with $n=4$
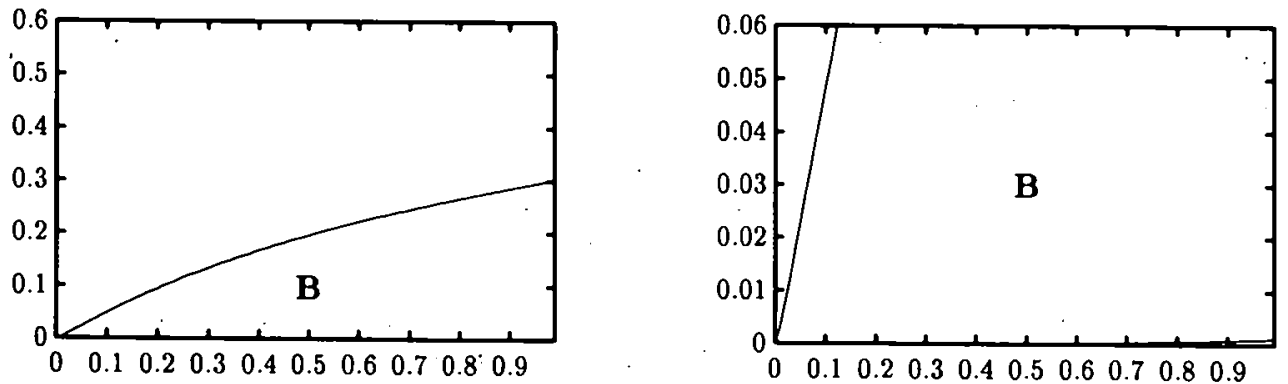

Figure 4: $\left(\frac{t}{T}, \frac{\delta}{E}\right)$-range $B$ of optimality for method (4.5)
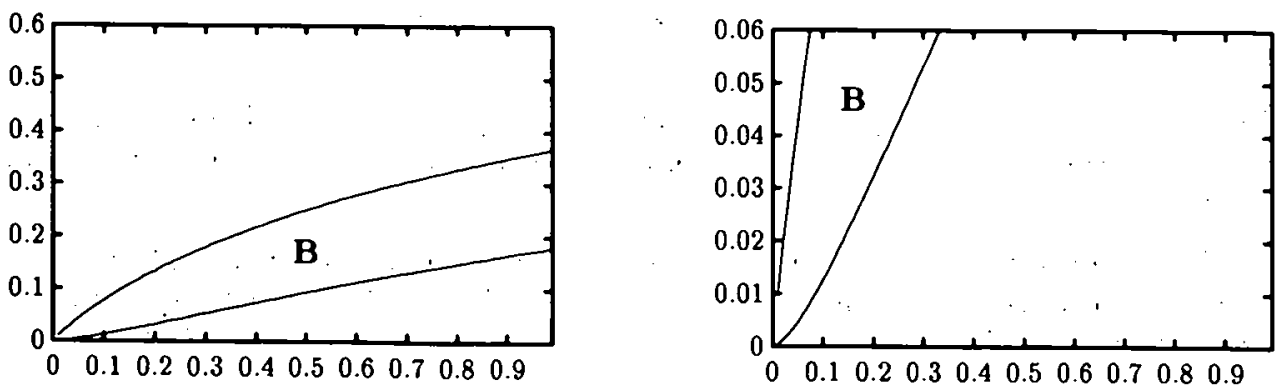
In the preceeding considerations we have treated the 'stationary' case, i.e. we have worked with a fixed $\alpha$-value in (4.7) (or (4.5), respectively). If the solution $q(t)$ is searched not only for one fixed $t \in[0, T)$; but for all $t \in[0, T)$, then one could apply (4.7) with the parameter choice (5.2) for a series of $t$-values $0=t_{0}<t_{1}<\ldots<t_{n}<T$ which would require to apply method (4.7) $n+1$ times with $n+1$ different $\alpha$-values. From the point of computational amount it seems to be much better to solve (4.7) (with $t=0$ ) only once and to work with a time-dependent regularization parameter. In this dynamical quasireversibility method a regularized approximation $q_{\beta}^{\delta}(t)(t \in[0, T))$ for equation (1.3) is obtained by solving (backward in time) for a given integer $n \geq 2$ the problem

$$
\left.\begin{array}{rl}
u_{t}(t)+A u(t)-\beta(t) A^{n} u(t) & =0 \quad \text { for } \quad 0 \leq t<T \\
u(T) & =z^{\delta}
\end{array}\right\}
$$

where $\beta(t)$ denotes the 'dynamical' regularization parameter. The solution of problem (5.6) can be expressed by

$$
q_{\beta}^{\delta}(t)=\sum_{i=1}^{\infty} e^{\lambda_{i}(T-t)-\lambda_{i}^{n} \int_{l}^{T} \beta(s) d s}\left(z^{\delta}, u_{i}\right) u_{i}
$$

where $\lambda_{i}$ are the eigenvalues and $u_{i}$ the eigenelements of the partial differential operator $A$. Consequently, the solution $q_{\beta}^{\delta}(t)$ of problem (5.6) coincides with the solution $q_{\alpha}^{\delta}(t)$ of problem (4.7) if $\alpha$ and $\beta$ are related by $\alpha^{n-1}(T-t)=\int_{t}^{T} \beta(s) d s$, which, for the parameter choice (5.2), is equivalent to

$$
\left[\frac{T}{\ln \frac{E}{\delta}}\right]^{n} \ln \frac{T}{t}=\int_{t}^{T} \beta(s) d s
$$

Now we differentiate (5.8), apply Theorem 5.1 and obtain

Theorem 5.3. Let $q_{\beta}^{\delta}(t)$ the regularized solution obtained by solving (5.6) backward in time with $n=2, n=3$ or $n=4$, respectively, where the dynamical regularization parameter $\beta(t)$ is chosen according to

$$
\beta(t)=\frac{1}{t}\left[\frac{T}{\ln \frac{E}{\delta}}\right]^{n} .
$$

Then, for the t-range given in Figure 1, Figure 2 or Figure 3, respectively; there holds the optimal error estimate

$$
\left\|q_{\beta}^{\delta}(t)-q(t)\right\| \leq E^{1-\frac{2}{T}} \delta^{\frac{t^{\prime}}{T}}
$$

Remark 5.4. The choice of the dynamical regularization parameter (5.9) in Theorem 5.3 guarantees that we have for the solution of the regularized problem (5.6) a well-posed propagation of the data $z^{\delta}$ 'backward in time' in such a way that optimality is preserved, i.e. for every $t$-value that belongs to the corresponding optimality range of Figure 1, Figure 2 or Figure 3 (in case $n=2, n=3$ or $n=4$, respectively) we can 
guarantee the optimal error bound (5.10), hence, the constructed approximation $q_{\beta}^{\delta}(t)$ is as accurate as possible in terms of the given information (A1) and (A2). For the choice of the integer $n$ in problem (5.6) we should prefer $n=2$ from the computational point of view. The larger $n$, the larger the computational amount for solving problem (5.6). ' On the other hand, the larger $n$, the larger the $t$-range of optimality for 'small' ratios $\frac{\delta}{E}$ (compare Figures $\left.1-3\right)$. Finally we note that the ratio $\frac{\delta}{E}$ can be influenced by incorporating an initial guess $\bar{q}$ for $q(0)$ into the solution process: let us define the regularized approximation $q_{\beta}^{\delta}(t)$ according to the following three steps:

(i) Given $\bar{q} \in H$, solve the well-posed 'forward problem'

$$
\left.\begin{array}{rl}
u_{t}+A u & =0 \quad \text { for } \quad 0<t \leq T \\
u(0) & =\bar{q}
\end{array}\right\}
$$

to obtain $u=\bar{u}(t)$ for $0 \leq t \leq T$.

(ii) Given $\bar{u}(T)$ from (i), solve the regularized problem (5.6) with $z^{\delta}$ replaced by $z^{\delta}-\bar{u}(T)$ to obtain the regularized approximation $u_{\beta}^{\delta}(t)$ for $t \in[0, T)$.

(iii) Given $\bar{u}(t)$ from (i) and $u_{\beta}^{\delta}(t)$ from (ii), compute the regularized approximation $q_{\beta}^{\delta}(t)=\bar{u}(t)+u_{\beta}^{\delta}(t)$ for $t \in[0, T)$.

Then the results of Theorem 5.3 remain true where the constant $E$ from assumption (A2) has to be replaced by a constant $E$ satisfying $\|q(0)-\bar{q}\| \leq E$.

\section{References}

[1] Baumeister, J.: Stable Solution of Inverse Problems. Braunschweig: Vieweg 1987.

[2] Engl, H. W.: Regularization methods for the stable solution of inverse problems. Surv. Math. Ind. 3 (1993), $71-143$.

[3] Gajewski, H. and K. Zacharias: Zur Regularisierung einer Klasse nichtkorrekter Probleme bei Evolutionsgleichungen. J. Math. Anal. Appl. 38 (1972), 784 - 789.

[4] Grigorieff, R. D. and R. Plato: On a minimax equality for seminorms. Lin. Alg. Appl. 221 (1995), $227-243$.

[5] Groetsch, C. W.: The Theory of Tikhonov Regularization for Fredholm Equations of the First Kind. Boston: Pitman 1984.

[6] Hanke, M. and P. C. Hansen: Regularization methods for large-scale problems. Surv. Math. Ind. 3 (1993), $253-315$.

[7] Hofmann, B. : Über Quelldarstellungen bei einigen lineareñ Regularisierungsverfahren. Beitr. Num. Math. 7 (1979), $75-81$.

[8] Hofmann, B.: Regularization for Applied Inverse and Ill-Posed Problems. Leipzig: B. G. Teubner Verlagsges. 1986.

[9] John, F.: Continuous dependence on data for solutions of partial differential equations with a prescribed bound. Comm. Pure Appl: Math. 13 (1960), 551 - 585.

[10] Lattès, R: and J. L. Lions: Méthode de quasi-réversibilité et applications. Paris: Dunod 1967. 
(11] Louis, A. K.: Inverse und schlecht gestellte Probleme. Stuttgart: Teubner 1989.

[12] Melkman, A. A. and C. A. Micchelli: Optimal estimation of linear operators in Hilbert spaces from inaccurate data. SIAM J. Numer. Anal. 16 (1979), 87 - 105.

[13] Miller, K.: Stabilized quasi-reversibilite and other nearly-best-possible methods for nonwell-posed problems. Lect. Notes Math. 316 (1973), $161-176$.

[14] Payne, L. E.: Some general remarks on improperly posed problems for partial differential equations. Lect. Notes Math. 316 (1973), $1-30$.

[15] Schröter, T. and U. Tautenhahn: On the optimality of regularization methods for solving linear ill-posed problems. Z. Anal. Anw. 13 (1994), 697 - 710.

[16] Seidman, T. I.: 'Optimal Filtering' for some ill-posed problems. In: Wave Propagation and Inversion (eds.: W. Fitzgibbon and M. Wheeler). Philadelphia: SIAM 1992, pp. 108 $-123$.

[17] Seidman, T. I.: Optimal filtering for the backward heat equation. SIAM J. Numer. Anal. (1995) (to appear).

[18] Tikhonov, A. N. and V. Y. Arsenin: Solution of Ill-Posed Problems. New York: Wiley 1977.

[19] Vainikko, G. M.: On the optimality of methods for ill-posed problems. Z. Anal. Anw. 6 (1987), $351-362$.

[20] Vainikko, G. M.: On the optimality of regularization methods. In: Inverse and Ill-Posed Problems (eds.: H. W. Engl and C. W. Groetsch). Orlando: Acad. Press 1987, pp. 77 95.

[21] Vainikko, G. M. and A. Y. Veretennikov: Iteration Procedures in Ill-Posed Problems (in Russian). Moscow: Nauka 1986.

Received 10.08.1995; in revised form 19.01.1996 